\title{
Warped geometry of brane worlds
}

\author{
Gary N Felder, Andrei Frolov and Lev Kofman \\ CITA, University of Toronto, 60 St George St, Toronto, Ontario, Canada M5S 3H8
}

Received 4 January 2002

Published 20 May 2002

Online at stacks.iop.org/CQG/19/2983

\begin{abstract}
We study the dynamical equations for extra-dimensional dependence of a warp factor and a bulk scalar in 5D brane world scenarios with induced brane metric of constant curvature. These equations are similar to those for the time dependence of the scale factor and a scalar field in 4D cosmology, but with the sign of the scalar field potential reversed. Based on this analogy, we introduce novel methods for studying the warped geometry. We construct the full phase portraits of the warp factor/scalar system for several examples of the bulk potential. This allows us to view the global properties of the warped geometry. For flat branes, the phase portrait is two dimensional. Moving along typical phase trajectories, the warp factor is initially increasing and finally decreasing. All trajectories have timelike gradient-dominated singularities at one or both of their ends, which are reachable in a finite distance and must be screened by the branes. For curved branes, the phase portrait is three dimensional. However, as the warp factor increases the phase trajectories tend towards the two-dimensional surface corresponding to flat branes. We discuss this property as a mechanism that may stretch the curved brane to be almost flat, with a small cosmological constant. Finally, we describe the embedding of branes in the 5D bulk using the phase space geometric methods developed here. In this language the boundary conditions at the branes can be described as a $1 \mathrm{D}$ curve in the phase space. We discuss the naturalness of tuning the brane potential to stabilize the brane world system.
\end{abstract}

PACS numbers: 1125,9880

\section{Introduction}

One of the most interesting recent directions in high energy physics phenomenology is the development of brane world scenarios in which our $(3+1)$-dimensional spacetime is a 3-brane embedded in a higher dimensional spacetime. In application to the very early universe this leads to brane world cosmology, where the universe we observe is a $(3+1)$-dimensional curved brane embedded in the bulk. One of the issues in brane world scenarios is the warped geometry of the internal space. In addition to the warp factor in the bulk, brane world scenarios often contain 
bulk scalar fields. Examples include the dilaton in Horava-Witten theory [1] (associated with the volume of the compactified 6D Calabi-Yau space) where the 5D effective theory can be obtained [2]; the Randall-Sundrum model [3] with phenomenological stabilization [5] where the choice of the bulk/brane potentials must be consistent with the $5 \mathrm{D}$ warp geometry $[6,7]$, the scalar sector of the supergravity realization of the Randall-Sundrum model [8], bulk supergravity with domain walls [10] and others.

The 5D bulk scalar plus gravity brane world system is based on the five-dimensional Einstein equations with junction conditions at the branes. Here we consider a simpler problem where the 5D space can be split into $(4+1)$ de Sitter slices

$$
\mathrm{d} s^{2}=\mathrm{d} w^{2}+A^{2}(w) \mathrm{d} s_{4}^{2} .
$$

The 4D de Sitter geometry is described by its scalar curvature ${ }^{4} R=12 H^{2}$, where $H=$ const is the 4D Hubble parameter. The warp factor $A(w)$ is determined up to boundary conditions by the five-dimensional Einstein equations. For the sake of generality we also present corresponding results for the more general case of a $D$-dimensional warped metric with $(D-1)$-dimensional de Sitter slices. The limit of vanishing $H$ corresponds to a flat brane, while non-vanishing $H$ corresponds to brane inflation.

The brane sets the boundary conditions for the warp factor $A(w)$ and the scalar field $\phi(w)$. It is known that often the warped geometry (1) somewhere outside of the brane encounters a spacetime singularity. One way to cure this problem is to invoke a second brane to screen the singularity by making the inner geometry periodic with the inter-brane interval. Two end-of-the-world branes provide orbifold compactification of the inner space. In the Randall-Sundrum model [3] with AdS bulk geometry without scalars the second brane may be removed.

The properties of warped geometry with one or two branes were studied in many papers, see e.g. $[6,7,9,10,12,13]$. The purpose of this paper is to investigate the global properties of 5D warped geometry (1) for a variety of bulk scalar field potentials $V(\phi)$, supplemented by boundary conditions at the branes. We will try to understand how typical is the singularity in the warped geometry, how much tuning is required for the brane potentials, and how these depend on the brane curvature $H^{2}$. Our approach is different from what was used in the earlier literature.

The setting of the problem for the geometry (1) is similar to the investigation of the FRW universe geometry

$$
\mathrm{d} s^{2}=-\mathrm{d} t^{2}+a^{2}(t) \mathrm{d} s_{3}^{2}
$$

with the scale factor $a(t)$ and a scalar field with the potential $V(\phi)$. A powerful method that has been used to investigate this $4 \mathrm{D}$ problem is the construction of phase portraits for the dynamic system for variables $\phi, \dot{\phi}$ and $\dot{a} / a$. Using this method it can be shown that for a broad range of potentials $V(\phi)$ inflation occurs along a separatrix that is a typical intermediate asymptotic for a broad band of phase trajectories [14, 15].

Inspired by this analogy, we adopt the phase portrait approach to studying the warped geometry of the brane world scenario. It turns out that the equations for the system $(A(w), \phi(w))$ with the potential $V(\phi)$ are similar to the cosmological equations for $(a(t), \phi(t))$ but with the sign of the potential reversed. (There are also differences in the numerical coefficients in 4D and 5D.) Flipping the sign of the potential makes a big difference. For example, it alters the geometry of the phase portrait by connecting branches with positive and negative 'Hubble' parameter $\frac{A^{\prime}}{A}$. This connection with 4D cosmology suggests a convergence of this work with recent work on $4 \mathrm{D}$ cosmology with negative potentials [16], the results of which can be extended to the warped geometry. Our results overlap with [16] and the connection will be investigated further [17]. 
The structure of the paper is the following. In section 2, we introduce the basic equations for the brane world scenario. In section 3, we discuss generic properties of the brane world phase space in terms of $\frac{A^{\prime}}{A}, \phi^{\prime}, \phi$ and classify its critical points.

In section 4, we systematically construct the phase portrait for a 5D space with flat 4D curvature $H=0$ (flat branes) for the simple quadratic potential $V(\phi)=\frac{1}{2} m^{2} \phi^{2}$. We will see that without branes all trajectories begin and end at naked singularities dominated by the gradient energy $\phi^{\prime 2}$ of the scalar field, which corresponds to a 'stiff' equation of state with anisotropic pressure. We also consider quadratic potentials with positive and negative cosmological constants.

In section 5, we consider exponential potentials $V(\phi)=V_{0} \mathrm{e}^{-2 \sqrt{2} \phi}$.

In section 6 , we extend the method of phase portraits to brane world scenarios with curved branes $H \neq 0$. We shall see that the brane with the larger warp factor will have smaller curvature. We discuss how this effect may be related to the problem of the small cosmological constant on the visible brane.

In section 7, we derive the Hamilton-Jacobi form of the self-consistent Einstein equations for warped geometry with a scalar field, which leads to the SUSY form of an arbitrary positive bulk scalar potential (without any underlying supersymmetry). This correspondence has been previously noted in the context of holographic renormalization group flows [11]. We also address the similarity of the Einstein-Hamilton-Jacobi constraint equation and the well-known gravitational stability form of the potential [10, 19, 20].

In section 8 , we introduce branes to screen the singularities. We show how the brane boundary conditions can be represented geometrically as a 1D curve in the 3D phase space of the system. It turns out to be convenient to use the EHJ formalism (in many respects similar to using the SUSY form of the potential). From this perspective we will discuss potentials that lead to brane stabilization and the degree of fine-tuning required to achieve them.

The paper concludes with the summary of our results. There is also an appendix in which the locations of critical points at infinity are derived for the phase portraits shown here.

\section{Equations and notation}

In this section we give the general formalism for a brane world scenario with a bulk warp factor and scalar field. The total action is

$S=\frac{1}{16 \pi \kappa_{D}^{2}} \int \sqrt{-g} \mathrm{~d}^{D} x\left\{R-(\nabla \phi)^{2}-2 V(\phi)\right\}-\frac{1}{8 \pi \kappa_{D}^{2}} \sum \int \sqrt{-h} \mathrm{~d}^{D-1} x\{[\mathcal{K}]+U(\phi)\}$.

The $D$-dimensional gravitational coupling is related to the $D$-dimensional Planck mass by $M_{D}^{D-2}=\frac{1}{8 \pi \kappa_{D}^{2}}$. The bulk scalar field $\phi$ in (3) is dimensionless. The physical value of the scalar field with canonical normalization is $\Phi=\frac{\phi}{\sqrt{8 \pi} \kappa_{D}}$ and the physical scalar field potential is $\frac{V}{8 \pi \kappa_{D}^{2}}$. The first term in (3) describes the bulk; the second term is related to the brane(s). We use a 'mostly positive' signature and the curvature conventions of Misner, Thorne and Wheeler (MTW). We write the jump of a quantity across the brane as $[\mathcal{K}]=\mathcal{K}^{+}-\mathcal{K}^{-}$. Bulk indices will be greek, $\mu, v, \ldots$; brane indices will be latin, $a, b, \ldots$. Brane hypersurfaces are denoted as $\Sigma_{i}$ where the index $i$ runs over all the branes. Throughout the paper we use overdots to indicate time derivatives and primes to indicate derivatives with respect to the fifth dimension $w$. 
The brane extrinsic curvature $\mathcal{K}_{a b}$ is expressed through the normal unit vector $n_{\mu}$ and the tangent vierbien $e_{(a)}^{\mu}$,

$$
\mathcal{K}_{a b}=e_{(a)}^{\mu} e_{(b)}^{\nu} \nabla_{\mu} n_{\nu},
$$

the bulk equations are

$$
R_{\mu \nu}-\frac{1}{2} R g_{\mu \nu}=T_{\mu \nu}, \quad \square \phi=\frac{\partial V}{\partial \phi},
$$

and the scalar field stress-energy tensor is given by

$$
T_{\mu \nu}=\phi_{, \mu} \phi_{, \nu}+\left(-\frac{1}{2}(\nabla \phi)^{2}-V(\phi)\right) g_{\mu \nu} .
$$

The junction conditions are

$$
\left[\mathcal{K}_{a b}-\mathcal{K} g_{a b}\right]=U(\phi) g_{a b}, \quad[n \cdot \nabla \phi]=\frac{\partial U}{\partial \phi} .
$$

For some brane world scenarios the bulk and brane scalar field potentials are known. In the case of the RS models, for example, we have $U(\phi)= \pm \lambda$, where $\lambda$ is a constant with different signs on the two branes. The bulk potential is just a $5 \mathrm{D}$ negative cosmological constant $V(\phi)=-\Lambda$. In case of the HW model, $U(\phi)=U_{0} \mathrm{e}^{-\sqrt{2} \phi}, V(\phi)=V_{0} \mathrm{e}^{-2 \sqrt{2} \phi}$. Our calculations will be valid for arbitrary $U(\phi)$ and $V(\phi)$.

For 5D warped geometry (1) the bulk Einstein equations can be written as

$$
\begin{aligned}
& \left(\frac{A^{\prime}}{A}\right)^{\prime}=-\frac{1}{4} \phi^{\prime 2}-\frac{1}{6} V(\phi)-\left(\frac{A^{\prime}}{A}\right)^{2}, \\
& 6\left(\frac{A^{\prime}}{A}\right)^{2}=\frac{1}{2} \phi^{\prime 2}-V(\phi)+6 \frac{H^{2}}{A^{2}},
\end{aligned}
$$

where the latter is a constraint equation. The equation for bulk scalar field

$$
\phi^{\prime \prime}+4 \frac{A^{\prime}}{A} \phi^{\prime}-V_{, \phi}=0
$$

is redundant with the Einstein equations.

In addition to the bulk equations given above we can specify boundary conditions at the brane. The assumption of $Z_{2}$ symmetry around each brane $\Sigma$ implies the boundary condition for the warp factor

$$
\left.\frac{A^{\prime}}{A}\right|_{\Sigma}=-\frac{1}{6} U
$$

and for the scalar field

$$
\left.\phi^{\prime}\right|_{\Sigma}=\frac{1}{2} U_{, \phi} .
$$

Unless otherwise specified all boundary conditions are given on the positive side of the brane.

When we refer to a flat or curved brane we are referring to its $4 \mathrm{D}$, i.e. spacetime, curvature. We only consider spatially flat branes, so the term flat brane refers to a $(3+1)$ Minkowski geometry while a curved brane refers to a $(3+1)$ de Sitter geometry.

The Einstein and field equations (8)-(10) can in principle be solved to find the $w$ dependence of $\phi$ and $A$, with boundary conditions supplied by the junction conditions (11) and (12). It is instructive to compare 5D warped geometry with a scalar field with 4D cosmological geometry (2) with a scalar field. In 4D cosmology we have the Einstein equations

$$
\left(\frac{\dot{a}}{a}\right)=-\frac{1}{3} \dot{\phi}^{2}+\frac{1}{3} V(\phi)-\left(\frac{\dot{a}}{a}\right)^{2}
$$




$$
3\left(\frac{\dot{a}}{a}\right)^{2}=\frac{1}{2} \dot{\phi}^{2}+V(\phi)-3 \frac{K}{a^{2}},
$$

where $K=0, \pm 1$ is the curvature of $3 \mathrm{D}$ space, plus a redundant scalar field equation

$$
\ddot{\phi}+3 \frac{\dot{a}}{a} \dot{\phi}+V_{, \phi}=0 .
$$

Apart from trivial numerical coefficients, equations (8)-(10) and (13)-(15) differ in only one respect: for the same sign of the potential in action (3) the sign of the potential in the cosmological equations will be opposite for the 4D cosmology and 5D brane world cases. The sign reversal comes about because of the different metric signatures of $\mathrm{d} w^{2}$ and $\mathrm{d} t^{2}$.

The 4D cosmological equations for positive potentials have been comprehensively studied by means of qualitative methods for analysing ODEs [14, 15]. These methods are equally applicable to 5D warped geometry. However, we will see below that the sign reversal has profound implications for the qualitative properties of the phase space behaviour.

Throughout the paper we will switch back and forth between a 4D and 5D viewpoint for the potentials we consider. We will usually talk about the shape of our potentials in terms of the behaviour they elicit. For example, we refer to the potential $V=\frac{1}{2} m^{2} \phi^{2}$ in 5D warped geometry (10) as a hill rather than a well, reflecting the fact that $\phi$ will tend to accelerate away from the origin rather than towards it for this case. The same potential acts as a well in the context of the 4D cosmological equation (15). We will examine the implications of our work for 4D cosmology with negative potentials in greater depth in a subsequent publication [17].

\section{Phase portraits}

The dynamical system (8)-(10) can be rewritten in terms of the variables

$$
x \equiv \phi, \quad y \equiv \phi^{\prime}, \quad z \equiv \frac{A^{\prime}}{A},
$$

which gives the three 'evolution' equations

$$
\begin{aligned}
& x^{\prime}=y \\
& y^{\prime}=V_{, x}-4 y z \\
& z^{\prime}=-\frac{1}{6} V-\frac{1}{4} y^{2}-z^{2},
\end{aligned}
$$

plus the constraint equation

$$
-2 V+y^{2}-12 z^{2}=-12 \frac{H^{2}}{A^{2}} .
$$

Here $V=V(x)$.

Reduction of equations to a set of first order ODEs allows us to represent their solutions using phase portraits, i.e. plots of trajectories in the 3D phase space defined by $x, y$ and $z$. This technique has been applied to the evolution equations for 4D chaotic inflation models in $[14,15]$. In general, all trajectories in phase space must begin and end at critical points, i.e. points where the derivatives of all the dynamic variables, i.e. the rhs of (17)-(19), vanish. In addition to starting and ending at such points, trajectories may in general come from or move towards infinity. By making a coordinate transformation of the phase space coordinates $(x, y, z)$ that project the complete phase space onto a compact region (Poincaré projection), however, one can define a discrete set of critical points at infinity. Together with the set of finite critical points these points describe the complete set of possible beginning and ending 
points for all trajectories. By identifying the properties of these critical points (attractors $A$, repulsors $R$ or saddle points $S$ ) and the trajectories that connect them it is possible to obtain a complete qualitative description of the dynamical system (17)-(19). (We do not expect more complicated situations for our dynamical system.)

Note that the parameter $H$ related to the curvature of the branes appears only in the constraint equation (20). Just as for standard FRW cosmology, our equations for warped bulk geometry can be classified by the sign of $H^{2}$. The case $H^{2}>0$ corresponds to curved branes with 4D de Sitter spacetime geometry, $H^{2}<0$ corresponds to 4D AdS spacetime, and $H=0$ corresponds to flat branes. These three cases correspond to three regions in phase space. Since $H^{2}$ is a constant of the motion, the phase space trajectories can never cross from one of these three regions to another. In particular this means that for any particular potential $V$ the surface obtained by setting $H^{2}=0$ in equation (20) defines a limiting surface in phase space that can never be crossed. In later sections we will see the importance of this two-dimensional surface for defining the phase space portrait for different potentials. In this paper we only consider trajectories in the regions with $H^{2} \geqslant 0$.

In the rest of this section we discuss finding the critical points for a general potential $V(x)$. In subsequent sections we illustrate this general procedure with a series of examples for which we construct the phase portraits.

To find the finite critical points we set all rhs of equations (17)-(19) to zero. This gives rise to the conditions

$$
\begin{aligned}
& y=V_{, x}=0, \\
& z^{2}=-\frac{1}{6} V(x) .
\end{aligned}
$$

For positive definite potentials there can be no finite critical points. For instance, for $V(\phi)=V_{0} \mathrm{e}^{-2 \sqrt{2} \phi}$ there are no finite critical points. For non-negative potentials finite critical points must all satisfy the conditions

$$
y=z=V=V_{, x}=0 .
$$

Any extremum for which $V<0$, however, corresponds to two critical points

$$
z= \pm \sqrt{-\frac{1}{6} V}
$$

To analyse the behaviour near these critical points $\left(x_{0}, y_{0}, z_{0}\right)$ we consider small deviations

$$
x=x_{0}+\delta x, \quad y=\delta y, \quad z=z_{0}+\delta z
$$

and linearize the equations (17)-(19). We then assume a solution of the form $(\delta x, \delta y, \delta z) \sim$ $\mathrm{e}^{\lambda w}$, which gives us a matrix with eigenvalues

$$
\lambda=\left(-2 z_{0},-2 z_{0} \pm \sqrt{V_{0, x x}+4 z_{0}^{2}}\right) .
$$

The negative eigenvalues correspond to attractors and positive eigenvalues to repulsors. Critical points whose eigenvalues have different signs are saddle points. Those with imaginary eigenvalues show oscillatory behaviour. In our case the finite critical points cannot be stable for $z_{0}<0$ or for $V_{0, x x}>0$. In either of these cases there will be at least one unstable direction in the vicinity of the critical point. For $z_{0}>0$ and $V_{0, x x}<0$ the solutions are stable.

It still remains to find critical points that occur at infinite values of the parameters. To do this we rescale the infinite space of $x, y$ and $z$ into a finite Poincare sphere by means of the variable definitions

$$
x=\frac{r}{1-r} \cos (\theta) \sin (\varphi)
$$




$$
\begin{aligned}
& y=\frac{r}{1-r} \sin (\theta) \sin (\varphi) \\
& z=\frac{r}{1-r} \cos (\varphi) .
\end{aligned}
$$

We also shall rescale $w$ by defining $\mathrm{d} \tilde{w}=\mathrm{d} w /(1-r)$.

Our phase space as described by the spherical coordinates $\{r, \varphi, \theta\}$ is contained within a sphere of radius 1 . The ODEs describing our system in these variables are written in the appendix. Here we are only concerned with critical points at infinity, which corresponds to $r=1$. In general the terms involving $V(x)$ and $V_{, x}(x)$ may be divergent for large $x$, in which case they alter the structure of the infinite critical points. Assuming $V$ and $V_{, x}$ are not divergent as $x \rightarrow \infty$, however, the set of infinite critical points turns out to be independent of the potential. Given this assumption, we found eight infinite critical points. We label them $\left(S_{1}, S_{2}, S_{3}, S_{4}, A_{1}, A_{2}, R_{1}, R_{2}\right)$, reflecting their behaviour for the potentials we have examined. ( $S \leftrightarrow$ saddle point, $A \leftrightarrow$ attractor, $R \leftrightarrow$ repulsor.) Their coordinates on the Poincaré sphere are at $r=1$ and

$$
\begin{aligned}
(\varphi, \theta)=(0,0) & ,(\pi / 2,0),(\pi / 2, \pi),(\pi, 0), \\
& \left(\sin ^{-1}(\sqrt{12 / 13}), \pi / 2\right),\left(\sin ^{-1}(\sqrt{12 / 13}), 3 \pi / 2\right), \\
& \left(\pi-\sin ^{-1}(\sqrt{12 / 13}), \pi / 2\right),\left(\pi-\sin ^{-1}(\sqrt{12 / 13}), 3 \pi / 2\right) .
\end{aligned}
$$

For the potentials considered in this paper several attractor and repulsor points are located on the two-dimensional surface corresponding to $H^{2}=0$.

Most realistic potentials are divergent as $x \rightarrow \infty$, with the result that infinite critical points can be added or removed relative to this generic picture. For instance, for $V=\frac{1}{2} m^{2} \phi^{2}$, $V=-\frac{1}{2} m^{2} \phi^{2}$, and $V=V_{0} \mathrm{e}^{-\phi}$ there are six, twelve and seven infinite critical points, respectively. Nonetheless, a close correspondence can usually be seen between the critical points for these potentials and the generic ones discussed here.

\section{Quadratic bulk potentials: $V=\frac{1}{2} m^{2} \phi^{2}$}

As a simple example of a bulk scalar potential we consider $V=\frac{1}{2} m^{2} \phi^{2}$. In the equations of motion the mass $m$ can be absorbed by rescaling the fifth coordinate $w \rightarrow m w$, so without loss of generality we simply set $m=1$. Thus in the general equations (17)-(19) we shall use

$$
V=\frac{1}{2} x^{2}, \quad V_{, x}=x .
$$

There is one finite critical saddle point for this case at the origin $x=y=z=0$. In the field equation (15) this point corresponds to the field sitting at the top of the potential with no velocity and the warp factor neither increasing nor decreasing. This state is, however, unstable. As we show in the appendix, there are only six infinite critical points for this case at $(\varphi, \theta)=(0,0),(\pi, 0),\left(\sin ^{-1}(\sqrt{12 / 13}), \pi / 2\right),\left(\sin ^{-1}(\sqrt{12 / 13}), 3 \pi / 2\right)$,

$$
\left(\pi-\sin ^{-1}(\sqrt{12 / 13}), \pi / 2\right),\left(\pi-\sin ^{-1}(\sqrt{12 / 13}), 3 \pi / 2\right) .
$$

In other words the set of infinite critical points is identical to the generic case (30) except without the points $(\pi / 2,0)$ and $(\pi / 2, \pi)$, i.e. $S 3$ and $S 4$ in figure 1 .

The constraint equation (20) for the quadratic potential has the form

$$
-x^{2}+y^{2}-12 z^{2}=-12 \frac{H^{2}}{A^{2}} .
$$

Setting $H^{2}=0$ in the constraint equation defines the two-dimensional surface

$$
-x^{2}+y^{2}-12 z^{2}=0
$$




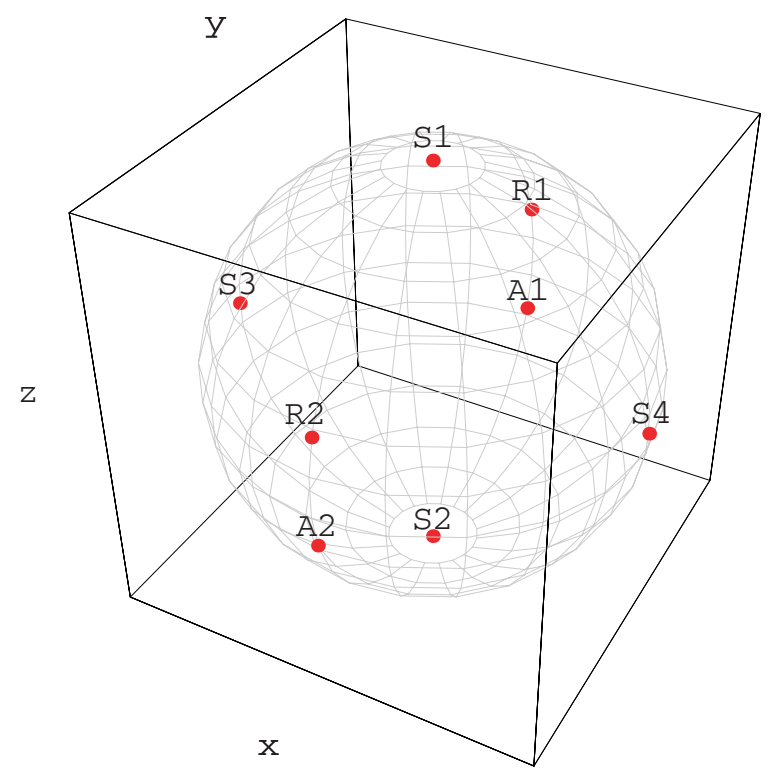

Figure 1. Infinite critical points on the Poincaré sphere. The labels are given to show correspondences with later plots for specific potentials.

which is a double cone opening up in the positive and negative $y$ directions, see upper panel of figure 2 .

The cases $H^{2}>0$ and $H^{2}<0$ correspond to trajectories outside and inside the double cone, respectively. For all values of $H^{2}$ nearly all trajectories begin at the points $R_{1}$ and $R_{2}$ on the top of the cone and end at the points $A_{1}$ and $A_{2}$ on the bottom, see figures 2 and 5. There are separatrices that connect saddle infinite points $S_{1}, S_{2}$ with the saddle point $S$ at the origin, see figure 5 .

In this section we consider trajectories that lie along the cone, i.e. 'flat' geometry with $H^{2}=0$. As we have mentioned, this case plays a special role in the investigation of the 3D phase portrait. We consider the case $H^{2}>0$ in section 6. Figure 2 shows Poincaré projection of two-dimensional phase space and some of the trajectories for the flat case. Phase trajectories that begin on the double cone surface (34) remain on it. Trajectories that lie off the cone can, and in general do, approach it in the limit $r \rightarrow 1$, but any trajectory that lies on the cone at any finite point must lie entirely on it. Therefore, the phase space required to describe warped bulk geometry with flat 4D slices is two dimensional.

The flat case we are considering is similar to 4D FRW flat cosmology with a scalar field, which can also be described with a two-dimensional phase portrait $[14,15]$. In the cosmological case the phase manifold has two disconnected planes, one for an expanding universe and another for a contracting universe (which can be obtained from the first by time reversal). In the $5 \mathrm{D}$ case, however, the expanding and contracting regions of the phase portrait are connected, which leads to very different behaviour of $A(w)$ and $\phi(w)$.

In addition to showing the $3 \mathrm{D}$ phase portrait, figure 2 also shows the projection of one of the cones onto the $(x, z)$ plane and the projection of the upper half of both cones onto the $(x, y)$ plane. There are several interesting features to note about this portrait. As in the 4D case, trajectories cannot pass from one cone to the other. Trajectories cannot pass through the critical point $S$ at the origin, or through any critical point for that matter, because by definition 

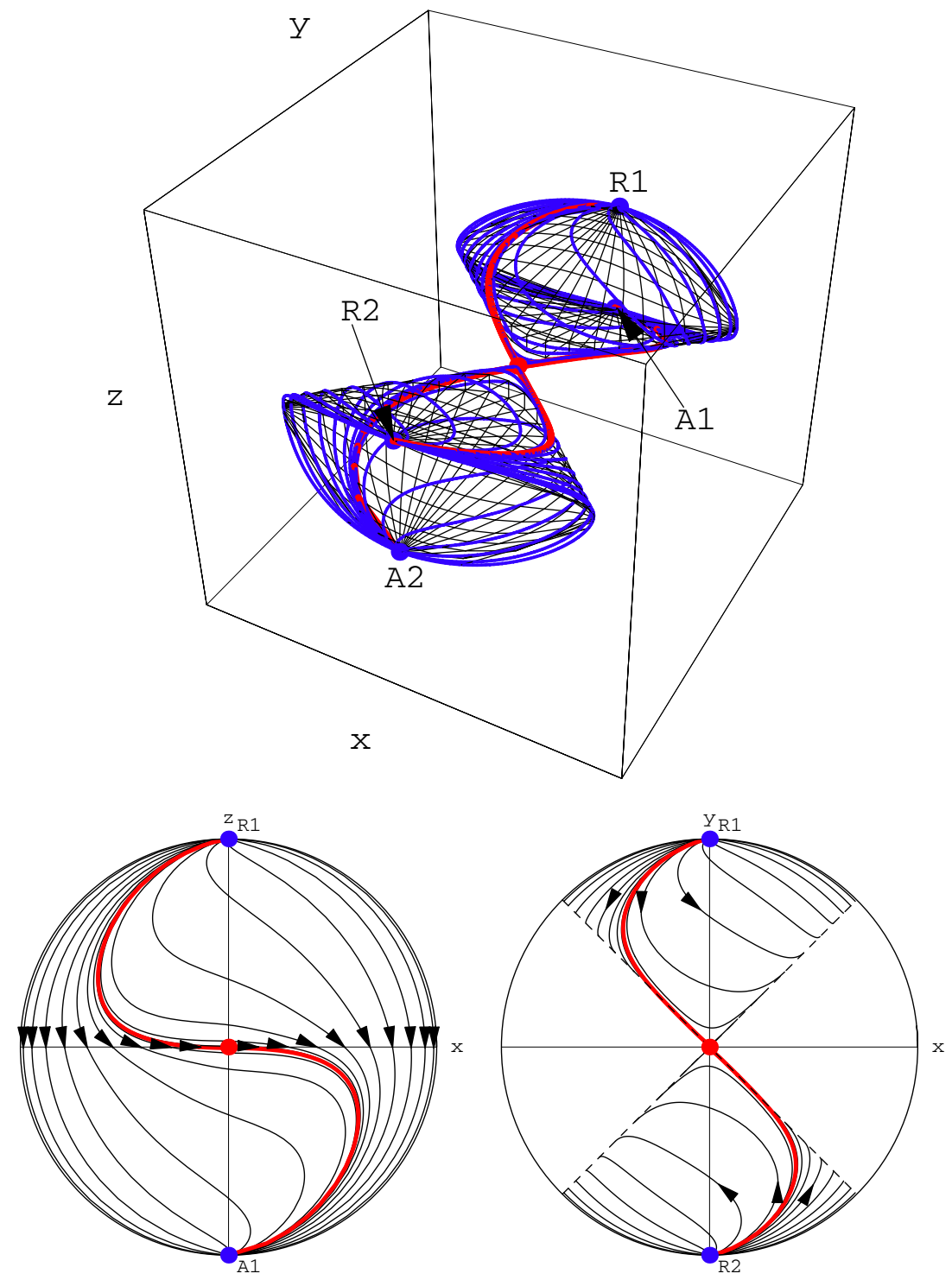

Figure 2. Phase portrait in Poincaré coordinates and trajectories with $H^{2}=0$ for $V=\frac{1}{2} m^{2} \phi^{2}$. The top figure shows the trajectories in the full 3D phase space while the bottom pictures show projections onto the $x z$ and $x y$ planes. In both projections, only half of the cone is shown for clarity. Trajectories at the $x y$ plane continue on the other side of the cone.

all derivatives vanish at these points. In the 4D case this separation meant that for $H^{2}=0$ with this scalar potential the sign of the Hubble parameter could never change. In the 5D case, by contrast, the geometry of the cone dictates that the sign of $y$, i.e. of $\phi^{\prime}$, can never change. Consider what this means for the equation of motion (10), which describes an inverted harmonic potential with friction

$$
x^{\prime \prime}+4 \frac{A^{\prime}}{A} x^{\prime}-x=0 .
$$


Ordinarily we would expect that if you start on one side of the hill $-x^{2}$ rolling towards the origin you could either go over the top or stop and roll back in the direction you came from. However, the latter behaviour is not possible for our dynamical system. The reason comes from the behaviour of the effective 'friction' $\frac{A^{\prime}}{A}=z$. Looking at the constraint equation (34) we can see that as $y$ decreases $z^{2}$ approaches zero. Once the kinetic energy is small enough $z$ will change sign. This means that the warp factor $A(w)$, which was initially increasing, will begin shrinking. At this turn-around point the friction term in the scalar field equation of motion (35) becomes negative, $y$ begins to grow again, and the field inevitably makes it over the top of the hill. From that point onward $y$ grows with increasing speed, eventually reaching the singular point at infinity within a finite distance. (If we insert brane at some $w_{1}$ so that $A(w)$ was decreasing initially then friction will always be negative, and the same result holds.)

This behaviour is clear in the phase portraits shown here. All trajectories must cross from $z>0$ to $z<0$, and must end up at the infinite critical point with $\frac{x}{y} \rightarrow 0$. In other words, as $\phi$ grows, $\phi^{\prime}$ grows infinitely faster, so the singularity is reached in a finite distance. Note that on each branch of the cone $( \pm y)$ there are two topologically distinct sets of trajectories, distinguished by which side of the cone $( \pm x)$ they are on when they cross from $z>0$ to $z<0$. Physically this difference simply reflects whether they crest the top of the hill before or after the turn-around point discussed earlier. These two classes of trajectories are separated by separatrices connecting the infinite critical points to the origin, as can be seen in the right-hand side of figure 2 .

All trajectories begin on the repulsive infinite critical points $R_{1}$ and $R_{2}$ where $z>0$ and end at the attractive infinite critical points $A_{1}$ and $A_{2}$ where $z<0$. This is why eventually $A(w)$ always decreases. The separatrix trajectories are obtained from two curves that intersect at the origin, one each starting from $R_{1}$ and $R_{2}$ and ending at $A_{2}$ and $A_{1}$, respectively.

Near the end points of all trajectories $R_{1}, R_{2}, A_{1}$ and $A_{2}$ the gradient term $\frac{1}{2} y^{2}$ dominates over the potential term $\frac{1}{2} x^{2}$. Recall that the energy density of the scalar field from (6) is $\rho=\frac{1}{2} \phi^{\prime 2}+\frac{1}{2} m^{2} \phi^{2}$; its pressure is anisotropic: $P_{w}=\frac{1}{2} \phi^{\prime 2}-\frac{1}{2} m^{2} \phi^{2}$, while in the other three directions $P_{1,2,3}=-\frac{1}{2} \phi^{\prime 2}-\frac{1}{2} m^{2} \phi^{2}$. Thus, in the regime where gradient terms dominate, we have $T_{v}^{\mu}=-\rho \operatorname{diag}(+1,+1,+1,+1,-1)$. (Recall that we use the MTW convention for $T_{\nu}^{\mu}$ where $\rho=-T_{0}^{0}$.) The pressure is anisotropic, in the direction of the extra dimension it corresponds to a stiff equation of state $P_{w}=\rho$, while in the perpendicular direction it has vacuum-like equation of state $P_{1,2,3}=-\rho$. Compare this with a stiff equation of state in 4D scalar field cosmology near a spacelike singularity, i.e. $P=\rho[14,15]$. As we approach infinite values of $\phi^{\prime}$, we have $\rho \propto A^{-2(D-1)}$, in $5 \mathrm{D} \rho \propto A^{-8}$. This corresponds to a timelike singularity (say at $w=w_{0}$ ) as $A(w) \rightarrow 0$. Near singularities $\phi \propto \sqrt{\frac{D-2}{D-1}} \log \left(w-w_{0}\right), A(w) \propto\left(w-w_{0}\right)^{\frac{1}{D-1}}$. In 5D, $A(w) \propto\left(w-w_{0}\right)^{1 / 4}$. We conclude that the end points of all trajectories correspond to timelike singularities. For realistic models these singularities should be screened by branes.

To end this section we modify the quadratic potential by adding a cosmological constant $V=1 / 2 m^{2} \phi^{2}+\Lambda$, where $\Lambda$ may have either sign. Negative $\Lambda$ with no scalar (or equivalently $m=0$ ) corresponds to the Randall-Sundrum [3, 4] model. In other words we are considering the question of what happens to the RS model (say AdS with a single flat brane) if one adds a massive bulk scalar. As we will see, this model has the same gradient type naked singularities that we found for the simple quadratic potential.

Once again we take $m=1$ so $V=\frac{1}{2} x^{2}+\Lambda$. Since $\Lambda$ is not divergent as $x \rightarrow \infty$ the set of six infinite critical points is the same as for $V=1 / 2 m^{2} \phi^{2}$. To consider the finite critical 


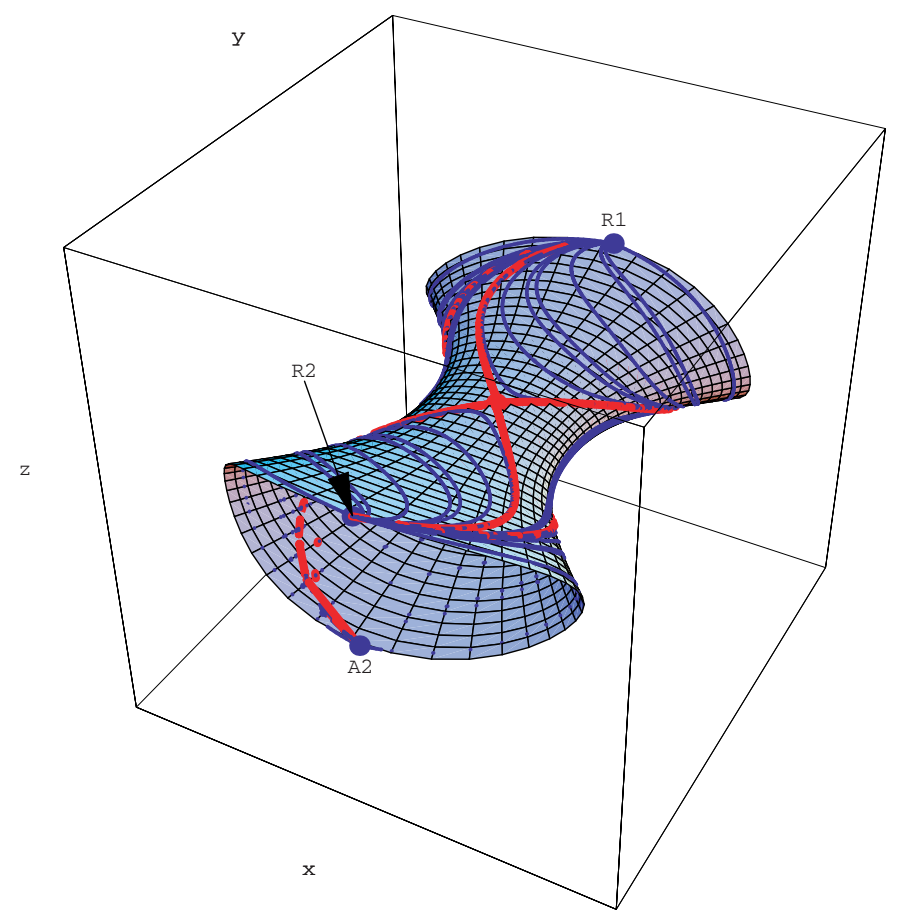

Figure 3. Trajectories with $H^{2}=0$ for $V=1 / 2 m^{2} \phi^{2}+\Lambda$ with $\Lambda<0$.

points and the limiting surface $H^{2}=0$, however, we have to distinguish between two cases based on the sign of $\Lambda$. The limiting surface is given by

$$
y^{2}=12 z^{2}+x^{2}+2 \Lambda,
$$

which is a hyperboloid of one or two sheets for $\Lambda<0$ and $\Lambda>0$, respectively. For $\Lambda>0$ the potential is positive definite and there are no finite critical points. For $\Lambda<0$ there are two finite saddle points at $x=y=0, z= \pm \sqrt{|\Lambda| / 6}$.

For $\Lambda>0$ the phase portrait is nearly identical to the case studied above. The only difference is that the two cones separate into two disconnected pieces of a hyperboloid. Since trajectories for $\Lambda=0$ could not pass from one cone to the other anyway, this change has no significant effect on the behaviour of the system.

Figure 3 shows the phase portrait for the quadratic potential with a negative cosmological constant. There are no longer two separated sheets, reflecting the fact that there are now trajectories connecting positive and negative $y$. There are two critical saddle points at which the field is sitting motionless at the top of the hill. There are eight separatrix trajectories, one each connecting each of the four infinite critical points to each of the finite critical points on the limiting surface. As before all trajectories begin and end at the $y$-dominated infinity. If we fix the brane at the middle of a trajectory, one of the singularities will be screened. However, without the second brane it is impossible to screen the other singularity. Therefore, the RS model with negative cosmological constant and a single $Z_{2}$ symmetric brane will acquire singularity at a final distance if a massive scalar field with non-vanishing condensate $\phi(w)$ is added. 

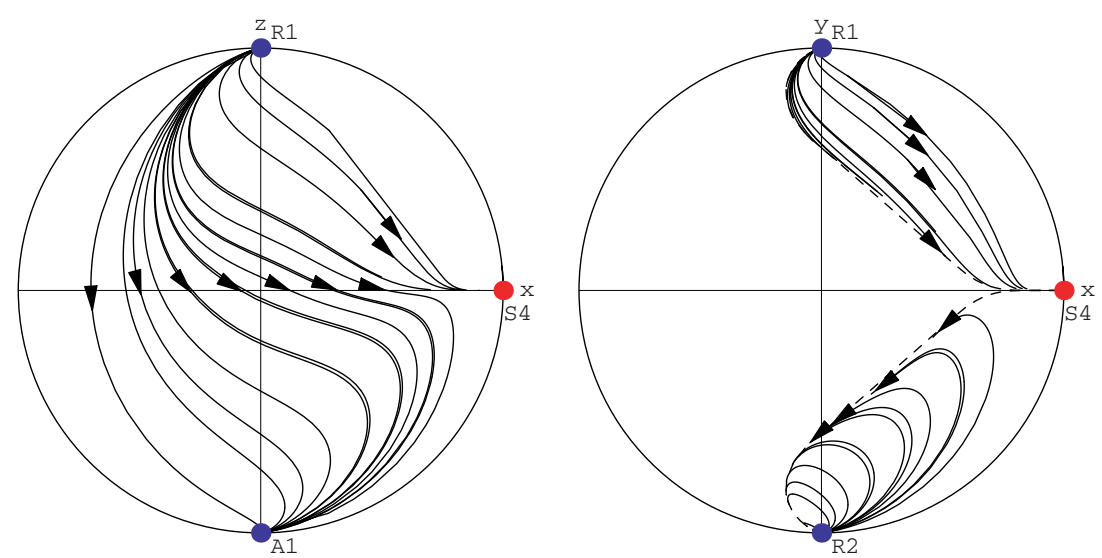

Figure 4. 2D projections of trajectories for $V=\mathrm{e}^{-2 \sqrt{2} \phi}, H^{2}=0$ on $x z(y>0)$ and $x y$ planes. In both projections, only half of the surface is shown for clarity.

\section{Exponential bulk potentials}

In this section we consider the bulk scalar potential $V=V_{0} \mathrm{e}^{-2 \sqrt{2} \phi}$. Dilaton scalar fields with exponential potentials naturally arise in many high dimensional theories. We consider the properties of warped geometry and dilaton using the phase portrait of their dynamical system. In our dimensionless variables, after rescaling of $w$ by $V_{0}^{1 / 2}$, we have $V=\mathrm{e}^{-2 \sqrt{2} x}$.

Since the potential is positive definite there are no finite critical points. Infinite critical points will not occur at any point where the potential diverges exponentially, i.e. for $x<0$. There are thus seven infinite critical points,

$$
\begin{gathered}
(\varphi, \theta)=(0,0),(\pi / 2,0),(\pi, 0),\left(\sin ^{-1}(\sqrt{12 / 13}), \pi / 2\right),\left(\sin ^{-1}(\sqrt{12 / 13}), 3 \pi / 2\right), \\
\left(\pi-\sin ^{-1}(\sqrt{12 / 13}), \pi / 2\right),\left(\pi-\sin ^{-1}(\sqrt{12 / 13}), 3 \pi / 2\right)
\end{gathered}
$$

corresponding to all points shown in figure 1 except $S_{3}$.

The limiting surface $H^{2}=0$ is described by

$$
y^{2}=12 z^{2}+2 \mathrm{e}^{-2 \sqrt{2} x} .
$$

As for the quadratic case, this surface consists of two branches, one each at positive and negative $y$, that touch only at a critical point. In this case that critical point is at $x=\infty$. For $H^{2}=0$ we once again have that $y$ can never change sign, meaning a field moving up the potential from negative infinity will always continue on to $x=+\infty$. There are, however, two very different ways this can occur. Figure 4 shows $2 \mathrm{D}$ projections of the phase portrait for this case. Consider upper half plane $y>0$. Many trajectories begin at infinite point $R_{1}$ $z>0, x / y \rightarrow 0$ and end at infinite point $A_{1} z<0, x / y \rightarrow 0$, just as they did for the quadratic potential. For these trajectories $y$ is growing ever more rapidly and a gradient singularity must occur at a finite distance (from the fixed brane). There is, however, another class of trajectories that approaches the infinite critical point $S_{4}$ at $z=y / x \rightarrow 0$. These are trajectories for which $y$ and $z$ asymptotically decrease as $x$ approaches infinity, so the field and metric do not diverge in a finite distance. There is no singularity at the end of trajectories which are heading towards $S_{4}$. Two types of trajectories are divided by a separatrix between $R_{1}$ and $S_{4}$. The behaviour for the $y<0$ half is similar.

All trajectories in figure 4 for the exponential bulk potential can be obtained analytically as closed form solutions of the Hamilton-Jacobi equation (56) derived in section 7. However, we 


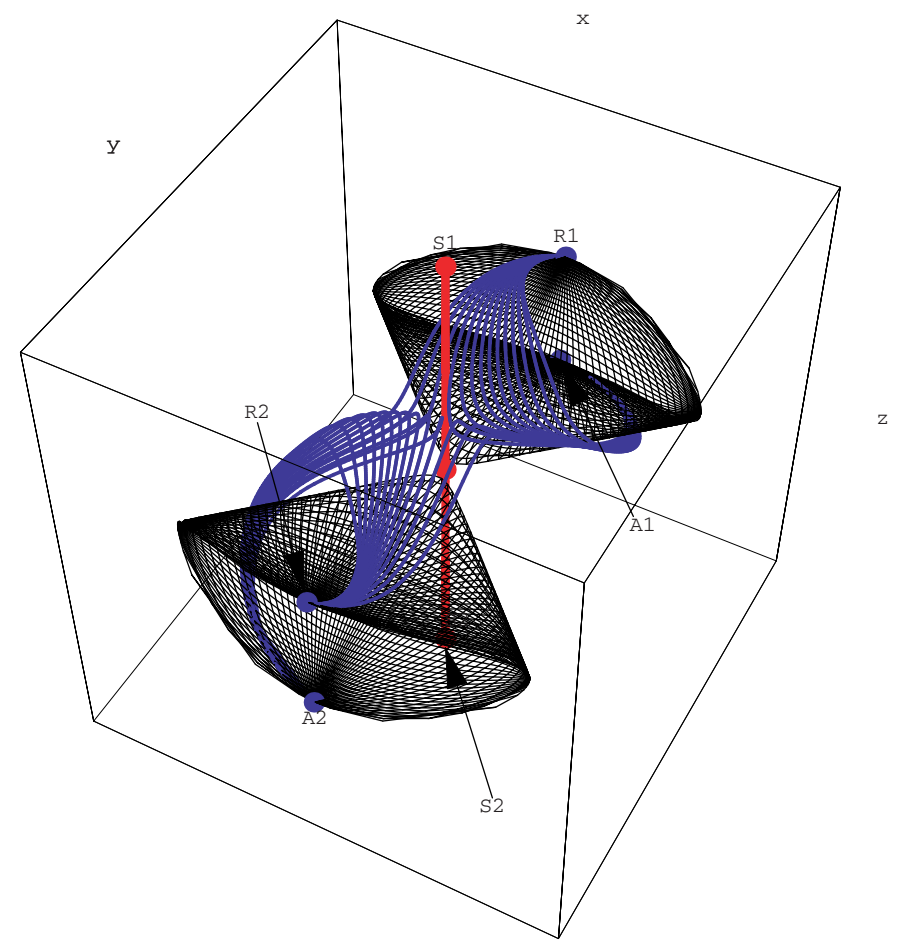

Figure 5. Trajectories with $H^{2}>0$ for $V=\frac{1}{2} m^{2} \phi^{2}$.

will not give their explicit form here, as the behaviour of the solutions is adequately described by the phase portrait already discussed.

\section{Warped geometry with 4D de Sitter slices}

In this section we extend the phase space analysis to include non-flat 4D sections of the 5D warped geometry with constant $4 \mathrm{D}$ curvature $\sim H^{2}$. We consider the case of positive $H^{2}$ corresponding to de Sitter 4D geometry. We continue to consider our major example, a simple quadratic potential $V(\phi)=\frac{1}{2} m^{2} \phi^{2}$, which we began to investigate in section 4 .

All trajectories in the 3D phase space $(x, y, z)$ in this case are located outside the cone. Without branes, a sampling of trajectories with $H^{2}>0$ is shown in figure 5 .

Once again, trajectories begin and end at the same four critical points on the top and bottom edges of the cone. Now, however, there is no topological constraint preventing $y$ from changing sign, so trajectories beginning at either of the points at $z>0$ can end at either of the points at $z<0$. As before, however, the warp factor always goes from increasing to decreasing as the derivative $A^{\prime} / A$ decreases monotonically from positive infinity to negative infinity. There is once again a topological constraint imposed by a pair of separatrices, however. In this case these are the trajectories that move straight down along the line $x=y=0$, one between the point $S_{1}$ and the origin $S$ and the other between the origin and the point $S_{2}$. Trajectories passing to the right of this line must end at $y>0$ and trajectories passing to the left of it must end at $y<0$. This constraint simply reflects the fact that if the field comes to rest on one side of the hill it will end up rolling down on that same side. Once again all trajectories end up with $x / y \rightarrow 0$, suggesting they reach the gradient type singularity in a finite distance. 
The most interesting feature of the flow of phase trajectories in three dimensions is that they all tend to lean towards the limiting surface of the cone in the region of positive $z$. The physical reason for this is the following. Inspecting the constraint equation (20) we see that for increasing $A(w)$ (positive $z=\frac{A^{\prime}}{A}$ ), the curvature term $\frac{H^{2}}{A^{2}}$ decreases. This means that the 4D de Sitter slices of the 5D geometry are getting flatter. This is very similar to how in 4D cosmology inflation flattens the universe. Imagine we fix two branes at $w_{1}$ and $w_{2}$, and the warp factor is increasing between them, $A\left(w_{2}\right)>A\left(w_{1}\right)$. Let us normalize $A\left(w_{1}\right)=1$. Let us rewrite the $5 \mathrm{D}$ metric, specifying the $4 \mathrm{D}$ de Sitter coordinates in the form

$$
\mathrm{d} s^{2}=\mathrm{d} w^{2}+A^{2}(w)\left(-\mathrm{d} t^{2}+\mathrm{e}^{2 H t} \mathrm{~d} \vec{x}^{2}\right) .
$$

The intrinsic 4D metric at the first brane at $w_{1}$ is

$$
\mathrm{d} s_{4}^{2}=\left(-\mathrm{d} t^{2}+\mathrm{e}^{2 H t} \mathrm{~d} \vec{x}^{2}\right)
$$

while the intrinsic metric at the second brane is

$$
\mathrm{d} s_{4}^{2}=A^{2}\left(w_{2}\right)\left(-\mathrm{d} t^{2}+\mathrm{e}^{2 H t} \mathrm{~d} \vec{x}^{2}\right) .
$$

Rescaling the $4 \mathrm{D}$ coordinates with $A\left(w_{2}\right), t^{\prime}=A\left(w_{2}\right) t, \vec{x}^{\prime}=A\left(w_{2}\right) \vec{x}$, we obtain 4D metrics in the canonical form

$$
\mathrm{d} s_{4}^{2}=-\mathrm{d} t^{\prime 2}+\mathrm{e}^{2 H^{\prime} t^{\prime}} \mathrm{d} \vec{x}^{\prime 2} .
$$

The physical curvature is described in terms of $H^{\prime}=H / A\left(w_{2}\right)$, which is smaller than $H$, and thus the second brane is flatter than the first one. The larger the $A\left(w_{2}\right)$, the flatter the brane at $w_{2}$. It would be interesting to apply this mechanism to the problem of smallness of the cosmological constant on the visible brane.

\section{Phase trajectories, Einstein-Hamilton-Jacobi equations and the SUSY form of the potential}

So far we have only considered warped geometry with a bulk scalar without including branes. Branes must be self-consistently embedded in the 5D spacetime in accordance with the junction conditions. The junction conditions involve the brane scalar field potential $U(\phi)$. Their solution is often found by using an (auxiliary) SUSY superpotential [6].

Before we apply our phase portrait methods to the brane world scenario with branes, we introduce one more element. Phase space trajectories can be conveniently described in terms of Hamilton or Hamilton-Jacobi equations. In general relativity, usually the ADM $(3+1)$ formalism is used to derive the Einstein-Hamilton-Jacobi equations. In the context of 4D FRW cosmology with a scalar field the Einstein-Hamilton-Jacobi equations were derived by Bond and Salopek [18]. In the context of holographic renormalization group flows, the Hamilton-Jacobi equations were considered by de Boer et al [11]. In this section we extend these results for a $D$-dimensional spacetime with a scalar field. We use $(D-1)+1$ splitting, but our $(D-1)$ hypersurface can be either timelike or spacelike, and we consider branes of arbitrary constant curvature, and not just the flat case. We find that for the flat brane geometry, the constraint equation reduces to the SUSY representation of the scalar potential (this occurs even though the system is not necessarily supersymmetric).

Let us consider an arbitrary $D$-dimensional metric in Gaussian normal coordinates,

$$
\mathrm{d} s^{2}=\epsilon \mathrm{d} w^{2}+g_{a b} \mathrm{~d} x^{a} \mathrm{~d} x^{b},
$$

where $\epsilon= \pm 1$ depending on the timelike or spacelike character of the $(D-1)+1$ splitting. For the 4D cosmological problem [18] $\epsilon=-1$, while for the 5D warped geometry $\epsilon=+1$. 
The components of the curvature tensor can be split according to the Gauss-Codazzi equations

$$
\begin{aligned}
& { }^{(D)} R^{a}{ }_{b c d}={ }^{(D-1)} R^{a}{ }_{b c d}+\epsilon\left(\mathcal{K}^{a}{ }_{d} \mathcal{K}_{b c}-\mathcal{K}^{a}{ }_{c} \mathcal{K}_{b d}\right) \\
& { }^{(D)} R^{w}{ }_{a b c}=\epsilon\left(\mathcal{K}_{a b: c}-\mathcal{K}_{a c: b}\right) \\
& { }^{(D)} R^{w}{ }_{a w b}=\epsilon\left(-\mathcal{K}_{a b, w}+\mathcal{K}_{a c} \mathcal{K}^{c}{ }_{b}\right) .
\end{aligned}
$$

The Einstein and Ricci tensor components can be split as

$$
\begin{aligned}
& { }^{(D)} G^{w}{ }_{w}=-\frac{1}{2}{ }^{(D-1)} R+\frac{\epsilon}{2}\left(\mathcal{K}^{2}-\mathcal{K}_{a b} \mathcal{K}^{a b}\right) \\
& { }^{(D)} G^{w}{ }_{a}=\epsilon\left(\mathcal{K}_{a}{ }^{c}: c-\mathcal{K}_{: a}\right) \\
& { }^{(D)} R_{a b}={ }^{(D-1)} R_{a b}+\epsilon\left(2 \mathcal{K}_{a}{ }^{c} \mathcal{K}_{b c}-\mathcal{K K}_{a b}-\mathcal{K}_{a b, w}\right) .
\end{aligned}
$$

We further specify the metric ansatz in a form unifying equations (1) and (2)

$$
\mathrm{d} s^{2}=\epsilon \mathrm{d} w^{2}+A^{2}(w) \gamma_{a b}\left(x^{i}\right) \mathrm{d} x^{a} \mathrm{~d} x^{b},
$$

where $\gamma_{a b}$ is the metric of $(D-1)$-dimensional constant curvature space, and we write

$$
\mathcal{H}=\frac{A^{\prime}}{A} \text {. }
$$

Although this function of the warp factor was already used for the 'Hubble' parameter $z$, here we have denoted it differently since the very same combination can be considered as a function of $\phi$ and will play the role of the Hamiltonian.

For this metric ansatz, we have

$$
\begin{array}{ll}
\mathcal{K}_{a b}=\frac{1}{2} g_{a b, w}=\mathcal{H} g_{a b}, & \mathcal{K}=(D-1) \mathcal{H}, \\
\mathcal{K}_{a b} \mathcal{K}^{a b}=(D-1) \mathcal{H}^{2}, & \mathcal{K}_{a b, w}=\left(\mathcal{H}^{\prime}+2 \mathcal{H}^{2}\right) g_{a b} .
\end{array}
$$

The bulk Einstein equations give us three equations. The first equation is

$$
-(D-2) \mathcal{H}_{, a}=\phi^{\prime} \phi_{, a} .
$$

It can be shown [18] that $\mathcal{H}=\mathcal{H}(\phi)$. Then from (49) we obtain the momentum constraint equation

$$
-(D-2) \mathcal{H}_{, \phi}=\phi^{\prime}
$$

The second equation is the energy constraint equation

$$
\frac{1}{2}(D-1)(D-2) \mathcal{H}^{2}-{ }^{(D-1)} R \frac{\epsilon}{2}=\frac{\phi^{\prime 2}}{2}-\epsilon\left[\frac{1}{2} \phi_{, a} \phi^{, a}+V(\phi)\right] .
$$

The third equation is

$$
{ }^{(D-1)} R_{a b}-\epsilon\left\{(D-1) \mathcal{H}^{2}+\mathcal{H}^{\prime}\right\} g_{a b}=\phi_{, a} \phi_{, b}+\frac{2}{D-2} V(\phi) g_{a b} .
$$

From the trace of the last equation and the energy constraint it follows that

$$
\frac{D-1}{2}\left(\mathcal{H}^{\prime}+\mathcal{H}^{2}\right)+\frac{\phi^{\prime 2}}{2}+\frac{\epsilon}{D-2} V(\phi)=0
$$

Next we suppose that $\phi=\phi(w)$. Then from (51)-(53) we find that the scalar field configuration in $D$ dimensions is described by a system of three variables $\left\{\phi, \phi^{\prime}, \mathcal{H}\right\}$

$$
\begin{aligned}
\mathcal{H}^{\prime} & =-\mathcal{H}^{2}-\frac{\phi^{\prime 2}}{D-1}-\frac{2 \epsilon}{(D-1)(D-2)} V(\phi), \\
\phi^{\prime \prime} & =\frac{\partial V}{\partial \phi}-(D-1) \mathcal{H} \phi^{\prime} .
\end{aligned}
$$

These equations generalize the equations (8)-(10) and (13)-(15). 
So far we have not specified the curvature of the $(D-1)$ hypersurfaces. Assuming that the induced brane world metric is de Sitter spacetime, ${ }^{(D-1)} R_{a b}=(D-2) \frac{H^{2}}{A^{2}} \gamma_{a b}$, and from (51) we obtain the constraint equation

$$
\epsilon V(\phi)=\frac{1}{2}(D-2)^{2}\left(\frac{\partial \mathcal{H}}{\partial \phi}\right)^{2}-\frac{1}{2}(D-1)(D-2)\left(\mathcal{H}^{2}-\epsilon \frac{H^{2}}{A^{2}}\right) .
$$

Let us apply the Hamilton-Jacobi constraint equation for warped geometry with $\epsilon=+1$ and flat $(D-1)$-dimensional slicing with $H^{2}=0$. In this case we have

$$
V(\phi)=\frac{1}{2}(D-2)^{2}\left(\frac{\partial \mathcal{H}}{\partial \phi}\right)^{2}-\frac{1}{2}(D-1)(D-2) \mathcal{H}^{2} .
$$

Here the scalar field potential is taken as a function of $\phi$ which is a solution of the self-consistent equations (in loose terminology, 'on-shell' value of the potential). It is not expected that the arbitrary potential $V(\phi)$ will have the SUSY form (56) for non self-consistent geometry.

Compare this equation with the well-known result that the stability of the bulk scalar field requires a 'supersymmetric' form of the potential $[19,20]$

$$
V(\phi)=2(D-2)^{2}\left(\frac{\partial W}{\partial \phi}\right)^{2}-2(D-1)(D-2) W^{2},
$$

where $W$ is some auxiliary function $W=W(\phi)$ which is called the 'superpotential', but which emerges from the requirement of stability even without supersymmetry.

Comparing equations (56) and (57), we see that the bulk potential (56) can be expressed in the SUSY form (57) where the Hamiltonian $\mathcal{H}$ plays the role of the superpotential $W=\frac{1}{2} \mathcal{H}$. It would be interesting to understand the relation between these two apparently different approaches to scalar field dynamics in $D$-dimensional spacetime that lead to such similar looking results. Here we have simply noted that the SUSY form of the potential is very convenient to treat the bulk and the junction conditions in a unified way.

The brane junction conditions (11), (12) in terms of the Hamiltonian $\mathcal{H}$ are

$$
-2(D-2) \mathcal{H}=U(\phi), \quad-2(D-2) \frac{\partial \mathcal{H}}{\partial \phi}=\frac{\partial U}{\partial \phi},
$$

where the values of all the functions are taken at the brane position $w$. Therefore, choosing $U(\phi)=-2(D-2) \mathcal{H}(\phi)$ at the brane, we automatically satisfy the junction conditions. This is similar to the approach of [6]. Here, however, we make an explicit connection between the superpotential $W$ and the Hamiltonian $\mathcal{H}$.

We also shall note that for curved branes with nonzero curvature $H^{2}$, the form (56) is not supersymmetric anymore.

\section{Warp geometry between branes}

In the previous sections we discussed how to solve the gravity/scalar field equations in the bulk. To complete the brane world picture we must include branes as well, which are embedded in the bulk according to junction conditions (11), (12). We will consider the case of static branes $\left(w_{i}=\right.$ const) only, and assume mirror symmetry across the brane ( $Z_{2}$ symmetry).

In the language of phase portraits, the two boundary conditions (11), (12) define a 1D curve in the 3D phase space. The equation for this curve is determined by the surface potential $U(\phi)$, and can be parametrized by the value of the scalar field $\phi$ as

$$
y= \pm \frac{1}{2} U_{, x}(x), \quad z=\mp \frac{U(x)}{6}
$$




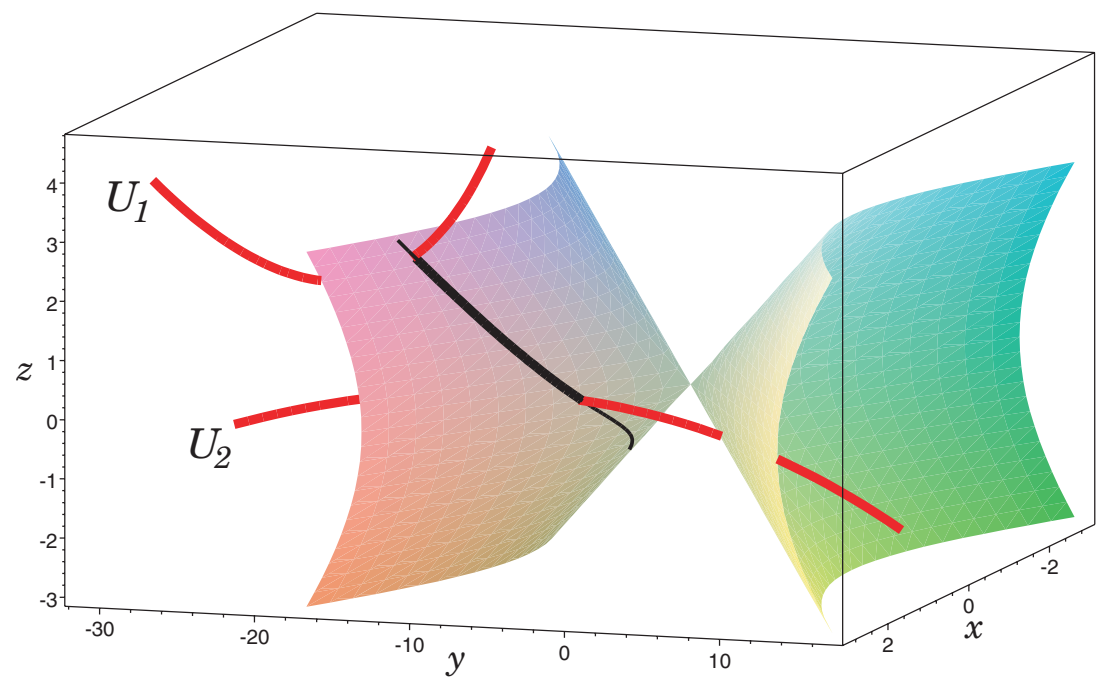

Figure 6. Segment of trajectory at the cone (shaded surfaces) in $\frac{1}{2} m^{2} \phi^{2}$ theory between two branes. Phase space positions of branes are obtained by intersection of the trajectory with $U_{1}, U_{2}$ curves, and is 'pinned' by the choice of brane surface potentials $U_{1}, U_{2}$.

where the signs correspond to the orientation (the choice of normal vector $n_{\mu}$ ) of the brane with respect to the bulk: the upper/lower signs are taken for the brane being on the left/right edge of the bulk, i.e. at the lower/higher limit of $w$. The bulk trajectories must start/end on these curves in the full phase space (and not at infinity as it was in the absence of branes) for junction conditions to be satisfied at the brane, as illustrated in figure 6 . The location of the brane is then given by the point of intersection of the phase space curve (59) and the bulk trajectory, and can be uniquely specified by the value of the field $\phi$ on the brane.

If one considers the case of a brane world with a single brane, for many potentials $V(\phi)$ one encounters a problem that, in general, singularities occur at a finite distance from the brane (although there are potentials such as exponential potential considered in section 6 , where singularity outside of $Z_{2}$ symmetric brane is avoidable). One possible solution to the problem of timelike singularity outside of the brane in the brane world scenario is to shield the singularity with a second brane. Given our assumption of $Z_{2}$ symmetry, the imposition of a second brane would effectively compactify the fifth dimension, making a circle with the two branes at opposite poles. The junction conditions must then be met separately at each brane, which is not necessarily guaranteed for an arbitrary choice of potentials $V$ and $U$.

Let us address the question about the existence of warped geometry bulk solution between two branes, if the potentials $U_{i}$ at the brane are arbitrary. It is known $[5,6]$ that in general such solutions can exist, but they are not expected to be compatible with the flat brane, but rather with a curved brane. We can obtain similar conclusion using the methods developed in this paper. To see this in terms of phase portraits, the problem of finding a consistent two-brane solution reduces to finding a trajectory in the full three-dimensional phase space that connects the two corresponding junction condition curves $U_{1}$ and $U_{2}$. Geometrically, this can be viewed as finding the intersection of a $2 \mathrm{D}$ surface generated by the bulk trajectories passing through the first curve $U_{1}$, and the second curve $U_{2}$.

As an intersection of a 2D surface and a curve in $3 \mathrm{D}$ is typically a point (unless they overlap or do not intersect altogether), for generic choice of $V$ and $U$ there may be only one 
such trajectory (if any), and the brane separation is fixed by its length. However, this successful trajectory is not expected in general to be located on the $H^{2}=0$ surface. Still, one can force the segment of trajectory between branes to be on the surface $H^{2}=0$ by simply shifting the potentials $U_{1}, U_{2}$ by a constant amount. This tuning is a familiar tuning of a four-dimensional cosmological constant on the brane (see also [6]).

We conclude that for potentials $U_{i}$ which are not specially selected, a brane world scenario is not expected to have flat branes. The branes in the self-consistent scenario are flat in a special but important case, which occurs when the junction condition curve is also a bulk trajectory, as happens for the Hamiltonian of the previous section. In this case, the two branes of opposite potential $U_{1}=-U_{2}=-2(D-2) \mathcal{H}$ can consistently be placed at any separation. If one modifies the brane surface potentials $U_{1,2}$ by adding quadratic corrections $\propto\left(\phi-\phi_{1,2}\right)^{2}$ as it was done in [6], the solution will be 'pinned' between $\phi_{1}$ and $\phi_{2}$, as illustrated in figure 6 , and the brane separation will be fixed by the choice of $\phi_{1}$ and $\phi_{2}$. In other words, the separation in the space of $\phi$ is translated into the inter-brane distance.

\section{Conclusion}

We have developed here a method for systematically exploring the properties of different potentials in brane world warped geometry. To construct the phase portrait of the dynamical system of gravity/scalar, one can apply the qualitative theory of differential equations. The solutions of these equations are represented by the trajectories propagating in the phase space. For a single bulk scalar, trajectories are in three-dimensional phase space. For the case of the flat branes, all trajectories are located at a two-dimensional surface, and the phase portrait of the dynamical system can be easily investigated.

In general, the phase space trajectories have timelike singularities at one or two of their ends. These singularities are dominated by the scalar field gradient term, and associated with the infinite critical points in the phase space. We describe how to find critical points for an arbitrary potentials. There are, however, examples of the potentials without singularity at one of the ends of the phase trajectory. In this case, it is possible to construct a non-singular warped geometry with a single brane with $Z_{2}$ symmetry.

We also considered the Einstein-Hamilton-Jacobi formulation of the warped geometry with scalar field. The constraint equation relates the arbitrary bulk potential, the Hamiltonian and its $\phi$-derivative. Surprisingly, the scalar potential taken on the self-consistent solutions acquires SUSY form even without underlying supersymmetry in the theory. We address the issue how this form of the constrain equation for arbitrary 'on-shell' potential is related to the requirement of the SUSY form of the potential for gravitational stability of a gravity/scalar system.

One can use the phase space with bulk trajectories to study warped geometry between two branes. Junction conditions for each brane generate a one-dimensional curve in the phase space. The segment of trajectory between two such curves corresponds to the inter-brane warp factor and scalar field. Without tuning the potential, this configuration in general is not located at the two-dimensional surface which represents the flat branes, in other words, the solution exists in general for curved branes. However, one can achieve solution with two flat branes by a simple shift of the potentials. This analysis can be easily extended for a more realistic case of several bulk scalar degrees of freedom. For instance, for two bulk scalars, the phase space is five dimensional and brane junction conditions generate a two-dimensional surface. Without tuning the brane potentials, in general there is a segment of trajectory which connects both two-dimensional surfaces in 5D (except for special cases). 
We leave the phenomenological applications of our methods for the construction of a brane world scenario with stabilization and investigation of their stability for future work.

\section{Acknowledgments}

We are grateful to Dick Bond, Andrei Linde, Renata Kallosh, Boris Khesin and Dario Martelli for fruitful discussions and comments. This work was supported by NSERC, CIAR, PREA of Ontario and NATO Linkage grant 975389.

\section{Appendix}

In this appendix we find the coordinates of the infinite critical points using the coordinates of the Poincaré mapping. We continue to denote derivatives with primes, with the understanding that all $r, \theta$ and $\varphi$ derivatives are with respect to $\tilde{w}$. In these new variables, our basic equations (17)-(19) for arbitrary potential become

$$
\begin{aligned}
r^{\prime}=\frac{1}{16}(1-r) & \cos \varphi\left(-25+9 \cos 2 \varphi+34 \cos 2 \theta \sin ^{2} \varphi\right) \\
+ & \frac{1}{8}(1-r)^{2}\left(4 \sin 2 \theta \sin ^{2} \varphi-\cos \varphi\left(-25+9 \cos 2 \varphi+34 \cos 2 \theta \sin ^{2} \varphi\right)\right) \\
& +\frac{1}{48}(1-r)^{3}\left(24 \sin \varphi\left(-2 V_{, x} \sin \theta+\sin 2 \theta \sin \varphi\right)\right. \\
& \left.+\cos \varphi\left(8 V+75-27 \cos 2 \varphi-102 \cos 2 \theta \sin ^{2} \varphi\right)\right), \\
\theta^{\prime}=-2 r \cos \varphi & \sin 2 \theta-(1-r) \sin ^{2} \theta+\frac{(1-r)^{2}}{r} V_{, x} \cos \theta \operatorname{cosec} \varphi, \\
\varphi^{\prime}=\frac{1}{32} r(-14 & \sin \varphi-18 \cos 2 \varphi \sin \varphi+13 \cos 2 \theta \sin \varphi+17 \cos 2 \theta \sin 3 \varphi) \\
& +\frac{1}{4}(1-r) \sin 2 \theta \sin 2 \varphi+\frac{(1-r)^{2}}{r}\left(V_{, x} \cos \varphi \sin \theta+\frac{1}{6} V \sin \varphi\right) .
\end{aligned}
$$

Suppose that the potential $V(x)$ and its derivative $V_{, x}$ are not divergent at infinity $x \rightarrow \infty$, and there is no 'accidental' cancellation of terms (see the example below). Taking the limit $r=1$ and putting the rhs of equations for $\theta^{\prime}, \varphi^{\prime}$ to zero, we find eight solutions for $\theta, \varphi$ at the Poincaré sphere, given by equation (30).

For the quadratic potential $V(x)=\frac{1}{2} x^{2}$. We plug the expressions for $V$ and $V_{, x}$ into the above dynamical equations. The behaviour of $r^{\prime}$ and $\theta^{\prime}$ at $r=1$ are unchanged. The equation for $\varphi^{\prime}$, however, picks up another nonzero term in that limit, namely

$$
\frac{1}{6} \frac{(1-r)^{2}}{r} V \sin \varphi=\frac{1}{12} \cos ^{2} \theta \sin ^{3} \varphi
$$

so the total $\varphi^{\prime}$ equation becomes

$$
\varphi^{\prime}=\frac{1}{96}\{(-39-54 \cos 2 \varphi+42 \cos 2 \theta) \sin \varphi+(50 \cos 2 \theta-1) \sin 3 \varphi\} .
$$

The rhs of equations for $\theta^{\prime}, \varphi^{\prime}$ vanish for six points $(\theta, \varphi)$, given by equation (32). Two points from (30) disappear due to the cancellation by the special form of the potential $V$.

For the exponential potential we have

$$
\begin{aligned}
& V=\mathrm{e}^{-2 \sqrt{2} x}=\exp \left(-2 \sqrt{2} \frac{r}{1-r} \cos \theta \sin \varphi\right), \\
& V_{, x}=-2 \sqrt{2} \mathrm{e}^{-2 \sqrt{2} x}=-2 \sqrt{2} \exp \left(-2 \sqrt{2} \frac{r}{1-r} \cos \theta \sin \varphi\right) .
\end{aligned}
$$

At the infinite point with $x<0$ the potential diverges exponentially, and one of the eight critical points (30) disappear. The remaining seven points are given by equation (37). 


\section{References}

[1] Horava P and Witten E 1996 Heterotic and type I string dynamics from eleven dimensions Nucl. Phys. B 460 506-24 (Preprint arXiv:hep-th/9510209)

[2] Lukas A, Ovrut B A, Stelle K S and Waldram D 1999 Heterotic M-theory in five dimensions Nucl. Phys. B 552 246-90 (Preprint arXiv:hep-th/9806051)

[3] Randall L and Sundrum R 1999 A large mass hierarchy from a small extra dimension Phys. Rev. Lett. 83 3370-3 (Preprint arXiv:hep-ph/9905221)

[4] Randall L and Sundrum R 1999 An alternative to compactification Phys. Rev. Lett. 83 4690-3 (Preprint arXiv:hep-th/9906064)

[5] Goldberger W and Wise M 1999 Modulus stabilization with bulk fields Phys. Rev. Lett. $834922-5$ (Preprint arXiv:hep-ph/9907447)

[6] DeWolfe O, Freedman D, Gubser S and Karch A 2000 Modeling the fifth dimension with scalars and gravity Phys. Rev. D 62046008 (Preprint arXiv:hep-th/9909134)

[7] Gibbons G, Kallosh R and Linde A 2001 Brane world sum rules J. High Energy Phys. JHEP01(2001)022 (Preprint arXiv:hep-th/0011225)

[8] Van Proeyen A 2001 The scalars of $N=2, D=5$ and attractor equations Preprint arXiv:hep-th/0105158

[9] Chamblin H and Reall H 1999 Dynamic dilatonic domain walls Nucl. Phys. B 562 133-57 (Preprint arXiv:hepth/9903225)

[10] Skenderis K and Townsend P 1999 Gravitational stability and renormalization-group flow Phys. Lett. B 468 46-51 (Preprint arXiv:hep-th/9909070)

[11] de Boer J, Verlinde E and Verlinde H 2000 On the holographic renormalization group J. High Energy Phys. JHEP08(2000)003 (Preprint arXiv:hep-th/9912012)

[12] Flanagan E, Tye S-H and Wasserman I 2001 Brane world models with bulk scalar fields Phys. Lett. B 522 155-65 (Preprint arXiv:hep-th/0110070)

[13] Davis S 2001 Brane cosmology solutions with bulk scalar fields Preprint arXiv:hep-ph/0111351

[14] Kofman L A, Linde A D and Starobinsky A A 1985 Inflationary Universe generated by the combined action of a scalar field and gravitational vacuum polarization Phys. Lett. B 157 361-7

[15] Belinskii V A, Grishchuk L P, Zel'dovich Ya B and Khalatnikov I M 1985 Inflationary stages in cosmological models with a scalar field Zh. Eksp. Teor. Fiz. 89 346-60

[16] Linde A 2001 Fast-roll inflation J. High Energy Phys. JHEP11(2001)052 (Preprint arXiv:hep-th/0110195)

[17] Felder G, Frolov A, Kofman L and Linde A 2002 Cosmology with negative potentials Preprint arXiv:hep-th/0202017

[18] Salopek D S and Bond J R 1990 Nonlinear evolution of long wavelength metric fluctuations in inflationary models Phys. Rev. D 42 3936-62

[19] Boucher W 1984 Positive energy without supersymmetry Nucl. Phys. B 242282

[20] Townsend P 1984 Positive energy and the scalar potential in higher dimensional (super) gravity theories Phys. Lett. B 14855 